\title{
Importância da perimetria de dupla freqüência na detecção do glaucoma: rastreamento em funcionários de hospital público numa área urbana de São Paulo
}

\author{
The role of frequency doubling perimetry in the diagnosis of glaucoma:screening in \\ employees of a public hospital in an urban area of São Paulo
}

\author{
Geraldo Vicente de Almeida ${ }^{1}$ \\ Carmo Mandia Júnior² \\ Maurício Della Paolera² \\ Niro Kasahara ${ }^{2}$ \\ Cristiano Caixeta-Umbelino ${ }^{3}$ \\ Paula Boturão de Almeida ${ }^{4}$ \\ Ricardo Nunes Eliezer ${ }^{5}$ \\ Renato Klingelfus Pinheiros \\ Francisco Soares Seixas ${ }^{6}$ \\ Mauricio Flank ${ }^{3}$ \\ Eduardo Villaça Filho ${ }^{4}$ \\ Ralph Cohen ${ }^{7}$
}

Trabalho realizado no Departamento de Oftalmologia da Santa Casa de São Paulo e Faculdade de Ciências Médicas da Santa Casa.

Diretor do Departamento de Oftalmologia da Faculdade de Ciências Médicas da Santa Casa de São Paulo.

${ }^{2}$ Médico Assistente do Setor de Glaucoma do Departamento de Oftalmologia da Santa Casa de Misericórdia de São Paulo.

${ }^{3}$ Fellow do Setor de Glaucoma do Departamento de Oftalmologia da Santa Casa de Misericórdia de São Paulo.

${ }^{4}$ Médica(o) Voluntária(o) do Setor de Glaucoma do Departamento de Oftalmologia da Santa Casa de Misericórdia de São Paulo.

Médico Voluntário do Setor de Glaucoma do Departamento de Oftalmologia da Santa Casa de Misericórdia de São Paulo. Pós graduando, nível doutorado, pela Universidade de São Paulo.

${ }^{6}$ Médico voluntário do Departamento de Oftalmologia da UNIFESP.

7 Chefe do Setor de Glaucoma Departamento de Oftalmologia da Faculdade de Ciências Médicas da Santa Casa de São Paulo.

Endereço para correspondência: Ralph Cohen - Av. Angélica, 1814, conj. 1005 - São Paulo (SP)

CEP 01228-200 - E-mail: ralphcohen@uol.com.br

Recebido para publicação em 27.08.2003

Versão revisada recebida em 25.08.2004

Aprovação em 28.10.2004

Nota Editorial: Pela análise deste trabalho e por sua anuência na divulgação desta nota, agradecemos ao Dr. Rui Barroso Schimiti.

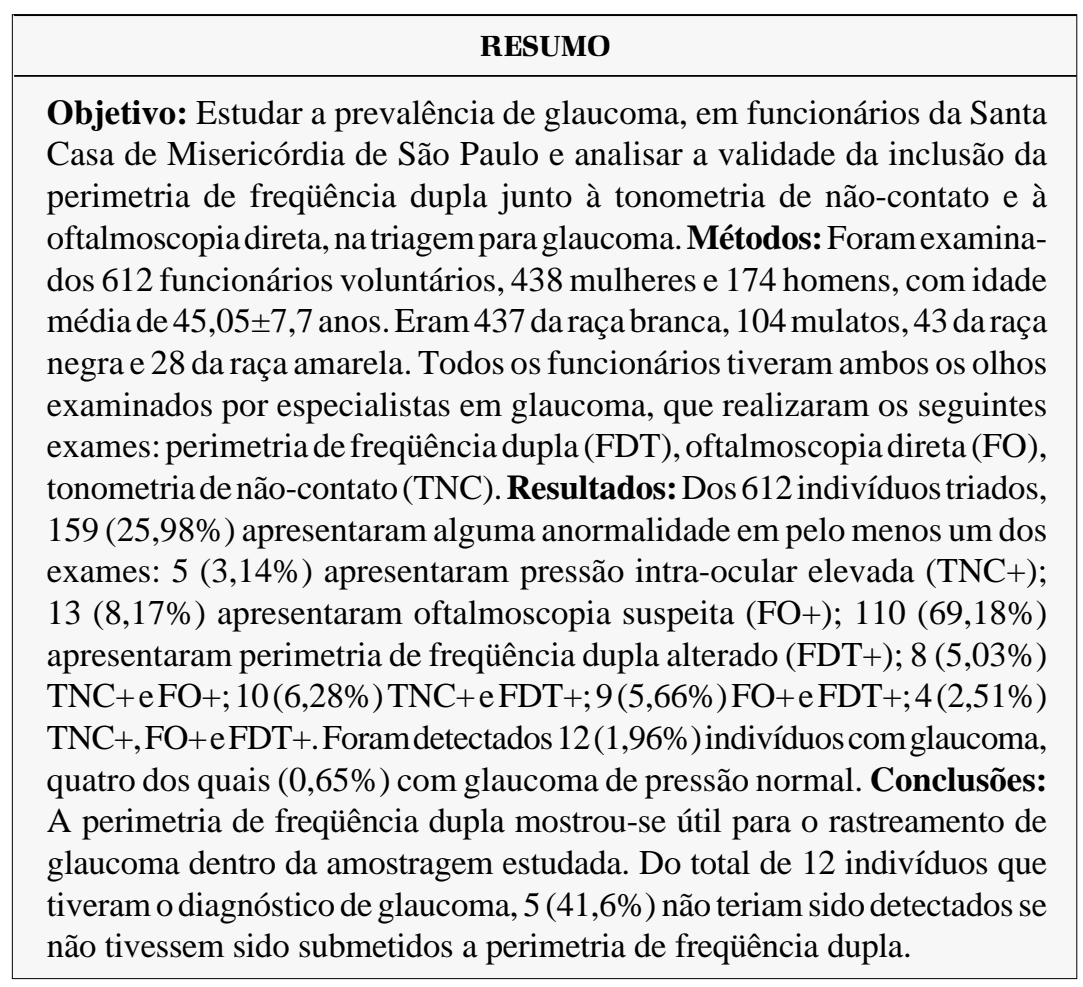

Descritores: Glaucoma/diagnóstico; Glaucoma/epidemiologia; Perimetria/métodos; Tonometria ocular; Oftalmoscopia

\section{INTRODUÇ̃̃̃O}

O glaucoma primário de ângulo aberto (GPAA) é uma enfermidade crônica, na qual ocorre lesão progressiva do nervo óptico, acompanhada de alterações características de campo visual. É considerada a segunda maior causa de cegueira no mundo. Estimou-se que no ano de 2000, aproximadamente 70 milhões de indivíduos seriam portadores dessa afecção e que cerca de $10 \%$ deles seriam cegos de ambos os olhos ${ }^{(1)}$. É uma doença insidiosa, em que sintomas são percebidos somente no estádio avançado de evolução, quando dano irreversível e acentuado já ocorreu. Observa-se então, a importância da conscientização da população quanto à necessidade de realização de exames que permitam seu diagnóstico precoce, principalmente em indivíduos com mais probabilidade de manifestá-la. 
Os programas de rastreamento populacional para glaucoma visam detectar novos indivíduos glaucomatosos e também informar ao público em geral dados sobre a enfermidade. Além disso, esses programas podem estimar a prevalência de glaucoma em diferentes comunidades.

A maioria dos programas de rastreamento usa a medida da pressão intra-ocular (PIO) como o exame primário deixando a avaliação do disco óptico e a perimetria para casos específicos. O relativo sucesso na identificação dos indivíduos glaucomatosos de fato é limitado pelo uso de apenas um ou dois métodos de exame ${ }^{(2-3)}$. A tonometria isolada tem sido o parâmetro mais utilizado e, tradicionalmente, a PIO maior do que $21 \mathrm{mmHg}$, como o valor tonométrico limite para caracterizar a hipertensão ocular. Entretanto, 50\% ou mais dos casos de glaucoma apresentam PIO inferior a essa medida nos rastreamentos, o que significa que essa medida isolada tem uma baixa sensibilidade para o diagnóstico da moléstia ${ }^{(4)}$.

A avaliação do disco óptico é um dos principais recursos para o diagnóstico do glaucoma, e quando realizada por indivíduos experientes, pode aumentar a sensibilidade da detecção do glaucoma ${ }^{(5-6)}$. Ainda assim, a validade para fins de rastreamento é questionada, uma vez que há grande variação quanto ao aspecto do disco óptico mesmo entre observadores treinados representando, da mesma forma, baixa sensibilidade para o diagnóstico ${ }^{(6)}$.

A perimetria automatizada acromática é um procedimento que necessita de muito tempo, não sendo prática para ser aplicada em rastreamentos populacionais.

Há evidências de que a sensibilidade ao contraste seja um parâmetro que se encontra alterado, nas fases iniciais, em portadores de hipertensão ocular e glaucoma ${ }^{(7)}$. A perimetria de freqüência dupla (FDT), idealizada com a finalidade de avaliar a sensibilidade de contraste, foi recentemente incorporada ao armamentário semiótico do glaucoma, por tratar-se de teste sensível, rápido e pouco oneroso ${ }^{(8)}$. Seu real valor ainda não está definido para o acompanhamento dos pacientes glaucomatosos, mas o seu uso já tem sido sugerido para fins de rastreamento ${ }^{(9)}$.

A maioria dos programas de triagem para o glaucoma apresenta falhas. O ideal seria que os rastreamentos incluíssem todos os parâmetros considerados "padrão ouro" para o diagnóstico do glaucoma, ou seja, tonometria de Goldmann, biomicroscopia do disco óptico com estereofotografia e perimetria computadorizada acromática. Entretanto, um programa com esse modelo torna-se inviável, tanto do ponto de vista prático quanto do financeiro.

Uma alternativa a ser testada seria a realização de triagem para o glaucoma envolvendo os três parâmetros básicos para o diagnóstico: avaliação da PIO, avaliação estrutural do disco óptico e avaliação funcional através da perimetria.

O presente estudo teve como objetivo estudar a prevalência do glaucoma, em funcionários da Santa Casa de Misericórdia de São Paulo e analisar a validade da inclusão da perimetria de frequiência dupla junto à tonometria de não-contato e à fundoscopia direta em triagens para o glaucoma.

\section{MÉTODOS}

O projeto da triagem para detecção de glaucoma foi submetido à avaliação e à aprovação da diretoria clínica e administrativa do Hospital Central da Santa Casa de Misericórdia de São Paulo. Foram examinados, de 15 a 20 de outubro de 2000, funcionários do Hospital, com idade superior a 35 anos, que se apresentaram voluntariamente para rastreamento de glaucoma, motivados por ampla divulgação interna da importância da detecção precoce do glaucoma, através de afixação de cartazes e de distribuição de folhetos explicativos, nos diferentes setores do Hospital. Os exames foram realizados em consultórios disponibilizados estrategicamente no saguão principal do Hospital.

O grupo estudado foi constituído de 612 indivíduos,

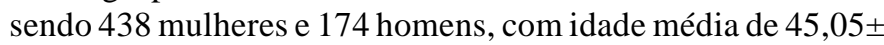
7,7 anos (variando de 35 a 81 anos). Quatrocentos e trinta e sete $(71,4 \%)$ indivíduos eram da raça branca, $104(17,0 \%)$ mulatos, $43(7,0 \%)$ da raça negra e $28(4,6 \%)$ da raça amarela.

O grupo de examinadores foi constituído de 10 oftalmologistas com experiência em glaucoma.

Todos os funcionários tiveram ambos os olhos examinados, iniciando o exame pelo olho direito, conforme o organograma (Figura 1):

A perimetria foi executada por técnico experiente, com o programa de "screening C20-1" do perímetro de frequiência dupla (Humphrey - FDT Visual Field Instrument, Humphrey - Dublin, Califórnia - EUA). Os pacientes usuários de lentes corretoras foram submetidos ao exame com a correção óptica, para longe.

A oftalmoscopia (FO) foi realizada com oftalmoscópio direto monocular Welch-Allyn, com a abertura de $5 \mathrm{~mm}^{2}$, sem instilação prévia de colírio midriático.

A tonometria (TNC) foi realizada com tonômetro de nãocontato (LEICA AT 550 - Reichert Ophthalmic Instruments, Buffalo, Nova Iorque - E.U.A.). Para cada olho foram realizadas três medidas consecutivas e para análise dos resultados foi considerada a média dessas três medidas.

Foram selecionados, na primeira fase do estudo, indivíduos nos quais foram encontrados pelo menos um dos seguintes parâmetros, em qualquer um dos olhos:

1) FDT+: redução da sensibilidade de contraste, com $p<5 \%$ em uma ou mais áreas nos $20^{\circ}$;

2) FO+: relação escavação/disco (c/d) $>0,5$ em um dos olhos ou assimetria >0,2, ou hemorragia no disco óptico, ou presença de diminuição localizada da rima neural sugestiva de glaucoma;

3) $\mathrm{TNC}+$ : $\mathrm{PIO} \geq 22 \mathrm{mmHg}$

De acordo com esses critérios, os indivíduos foram divididos em 7 grupos:

a) Grupo com TNC+;

b) Grupo com FO+;

c) Grupo com FDT+;

d) Grupo com TNC+ e FDT+;

e) Grupo com TNC + e FO +;

f) Grupo com $\mathrm{FO}+\mathrm{e} \mathrm{FDT}+$;

g) Grupo com TNC+, FO+e FDT+. 


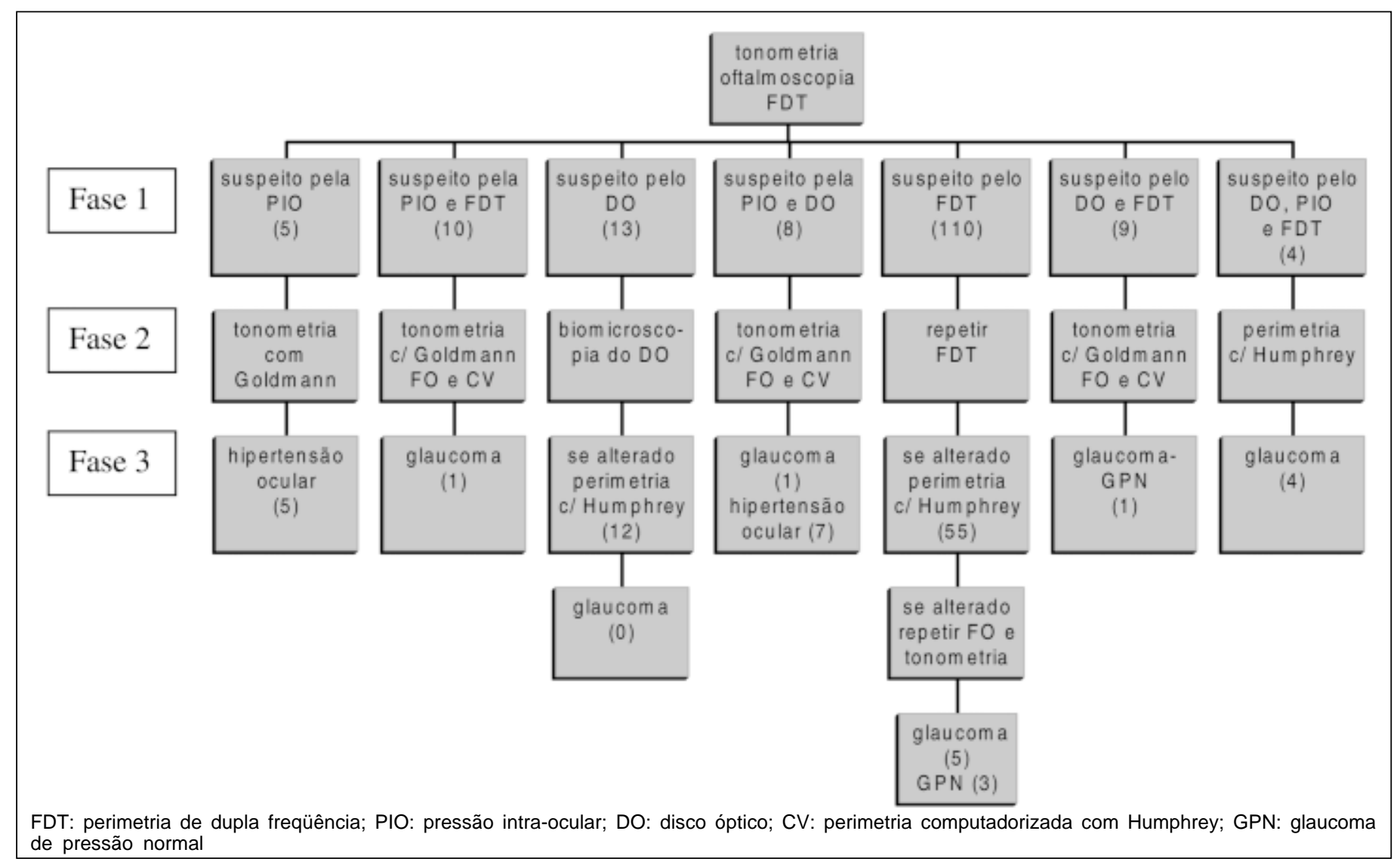

Figura 1 - Fluxograma de atendimento e número de casos ( $n$ ).

Na segunda fase do estudo, todos os indivíduos que apresentaram pelo menos uma área com $\mathrm{p}<5 \%$ no FDT (FDT + ), quer isoladamente ou em combinação com outro parâmetro, tiveram o exame de FDT repetido. Os indivíduos com PIO $\geq 22 \mathrm{mmHg}(\mathrm{TNC}+)$ foram submetidos à tonometria de Goldmann e os selecionados pelo aspecto suspeito do disco óptico foram submetidos à biomicroscopia de fundo com lente de Volk, sob midríase.

$\mathrm{Na}$ terceira fase, todos os indivíduos selecionados dos 7 grupos foram submetidos tanto à tonometria de Goldmann, como à biomicroscopia do disco óptico, sob midríase com lente de Volk 90D e também à perimetria computadorizada acromática com aparelho Humphrey 740 (Zeiss-Humphrey Instruments Inc., San Leandro, CA, EUA), aplicando-se o programa central 24-2 threshold (estratégia SITA) após refração automática. Para considerar anormalidade de campo visual, adotou-se a presença de pelo menos um dos seguintes critérios:

1) presença de três pontos adjacentes no gráfico "pattern daviation (PD)" com $\mathrm{p}<5 \%$, sendo um deles com $\mathrm{p}<1 \%$;

2) "glaucoma hemifield test (GHT)" fora dos limites normais;

3) "patern standard deviation (PSD)" com p <5\% ${ }^{(10)}$.

Neste estudo, como a gonioscopia não foi realizada, quando submetidos à biomicroscopia, quando nenhum sinal de glaucoma secundário esteve presente, o paciente foi rotulado como portador de glaucoma primário, sem diferenciação quanto à abertura do seio camerular.

Os resultados de prevalência foram calculados dividindose o número de indivíduos afetados pelo número total de indivíduos examinados.

\section{RESULTADOS}

Na primeira fase, 612 indivíduos foram triados, 159 (25,98\%) dos quais apresentaram pelo menos um teste anormal:

- $5(3,14 \%)$ apresentaram TNC+;

- $13(8,17 \%)$ apresentaram FO+;

- $110(69,18 \%)$ apresentaram FDT+;

- $8(5,03 \%)$ apresentaram $\mathrm{TNC}+\mathrm{e} \mathrm{FO}+$;

- $10(6,28 \%)$ apresentaram TNC+ e FDT+;

- $9(5,66 \%)$ apresentaram FO+ e FDT+;

- $4(2,51 \%)$ apresentaram TNC+, FO+ e FDT+

Assim, no grupo dos indivíduos com PIO $\geq 22 \mathrm{mmHg}$, isoladamente, todos os cinco indivíduos continuaram na mesma condição, após tonometria de Goldmann.

Todos os 13 indivíduos incluídos, especificamente, pelo aspecto do disco óptico, após biomicroscopia negativa do DO foram dispensados. Dos 110 indivíduos incluídos pelo FDT, cinco indivíduos faltaram ao reteste. Dos 105 reexaminados, 50 
tiveram FDT normal e foram dispensados e 55 mantiveram-se suspeitos. Desses, 39 compareceram para avaliação complementar (terceira fase). A perimetria revelou cinco indivíduos com lesão característica de glaucoma: três foram rotulados como portadores de glaucoma de pressão normal e dois como portadores de glaucoma primário.

O único indivíduo suspeito pela PIO e aspecto do FO que compareceu à terceira fase do estudo, apresentou perimetria normal, continuando, assim, na mesma condição de suspeição.

Dos dez indivíduos incluídos pela PIO e pela redução da sensibilidade de contraste (FDT + ), nove tiveram perimetria normal e mantiveram-se, também, na mesma condição de suspeição. Um único indivíduo apresentou lesão perimétrica glaucomatosa.

Dos nove suspeitos pelos aspectos do FO e FDT, oito tiveram exames perimétricos normais, tendo sido também colocados entre os suspeitos. Um paciente de 71 anos de idade com lesão perimétrica consistente foi considerado portador de glaucoma de pressão normal.

Os quatro indivíduos suspeitos pelos 3 parâmetros tiveram o diagnóstico de glaucoma primário e foram encaminhados para tratamento.

Assim sendo, nessa campanha foram detectados 12 $(1,96 \%)$ indivíduos com glaucoma, quatro $(0,65 \%)$ dos quais com glaucoma de pressão normal. Permaneceram como suspeitos, $54(8,82 \%)$ indivíduos.

\section{DISCUSSÃo}

Atualmente, o GPAA é definido como uma neuropatia crônica e progressiva, caracterizada por defeitos estruturais e funcionais do nervo óptico, independentemente dos níveis de PIO.

Apesar de a tonometria ser ainda um meio semiológico muito popular, sua importância como parâmetro único de diagnóstico precoce da doença tem sido reduzida, por falta de equilíbrio entre a sensibilidade e a especificidade, uma vez que, quando é adotado o nível de $21 \mathrm{mmHg}$ ou superior como critério de corte para suspeição da doença, a sensibilidade é de apenas $47,1 \%$ enquanto a especificidade é de $92,4 \%$. Por outro lado, quando se utiliza valor de Po igual ou inferior a $19 \mathrm{mmHg}$, a sensibilidade aumenta, porém a especificidade cai para aproximadamente $65 \%{ }^{(11)}$. Neste estudo, escolheu-se medir a PIO por meio de tonômetro de não-contato, por ser prático e rápido e bem indicado para rastreamentos populacionais ${ }^{(12)}$. Para otimizar a sensibilidade e a especificidade da tonometria de não-contato, utilizou-se a média de três medidas de PIO para cada olho examinado ${ }^{(13)}$.

A fundoscopia, por sua vez, pode oferecer maior sensibilidade e especificidade, desde que realizada por examinadores treinados e sob midríase. Neste estudo, todos os indivíduos estudados foram avaliados por especialistas em glaucoma. Entretanto, a instilação de colírio midriático para a realização de oftalmoscopia direta não foi realizada na avaliação inicial para não prejudicar a maioria dos funcionários, os quais esta- vam em horário de trabalho. Posteriormente, para a complementação do estudo (segunda e terceira fase), realizou-se a instilação de midriático com a finalidade de se avaliar o fundo de olho mais minuciosamente.

A inclusão de um teste de avaliação funcional do nervo óptico aumentaria, teoricamente, a sensibilidade da triagem. Alguns autores, realizando perimetria automatizada e tonometria identificaram $100 \%$ dos indivíduos glaucomatosos, ou seja, dez numa população de 145 indivíduos $^{(4)}$. Esses resultados, porém, podem ter sido influenciados por um viés, uma vez que a amostragem é muito pequena.

Estudos recentes demonstraram a utilidade do FDT como ferramenta útil no diagnóstico de dano funcional do nervo óptico, no glaucoma ${ }^{(14)}$. Johnson e Samuels chamaram a atenção para a rapidez de execução do exame, a boa confiabilidade no teste/reteste e a boa sensibilidade e especificidade na detecção de dano glaucomatoso ${ }^{(15)}$. É um exame que, dentre outros fatores, tem a vantagem de ser mais facilmente compreendido pelos pacientes que a perimetria automatizada convencional, quando aplicado em indivíduos que o realizam pela primeira vez, além de permitir a obtenção de resultados confiáveis em portadores de ametropias de até $7 \mathrm{D}$, sem o uso de qualquer correção óptica.

No presente estudo, $110(17,8 \%)$ funcionários foram considerados suspeitos exclusivamente pelo FDT. Dos 105 que compareceram no reteste, $50(47,6 \%)$ foram considerados normais. $\mathrm{O}$ grande índice de falso-positivos obtido pode ser explicado pelo efeito aprendizado. Dos 55 indivíduos cuja segunda avaliação pelo FDT confirmou o defeito, $31(56,4 \%)$ tiveram exame normal com a perimetria acromática. $\mathrm{O}$ acompanhamento desses indivíduos com posterior verificação do dano pela perimetria acromática poderá constituir evidência de diagnóstico precoce realizado pelo FDT. Do total de 110 suspeitos pelo FDT, cinco indivíduos tiveram o diagnóstico de glaucoma confirmado, três dos quais rotulados como glaucoma de pressão normal.

Nosso estudo mostrou uma baixa especificidade do FDT no diagnóstico de glaucoma. Dos 110 pacientes suspeitos exclusivamente pelo FDT, 55 revelaram-se normais após o reteste, e do restante cinco foram diagnosticados como glaucomatosos. Entretanto, do total de 12 indivíduos que tiveram o diagnóstico de glaucoma, cinco $(41,6 \%)$ deles não teriam sido detectados se não tivessem sido submetidos ao FDT. Portanto, embora pouco especifico, o FDT mostrou-se instrumento importante na triagem do glaucoma.

Diferente de um grande estudo populacional, este estudo teve como objetivo determinar apenas a prevalência de glaucoma através de uma campanha de triagem numa população específica, baseando-se na tonometria, na avaliação qualitativa do disco óptico (oftalmoscopia direta) e na avaliação funcional do nervo óptico (FDT e Humphrey). Qualquer exame complementar adicional, acarretaria em custos adicionais para sua execução. Todos os pacientes rotulados como glaucomatosos foram encaminhados para o serviço de oftalmologia do hospital onde foram submetidos a uma avaliação completa. 


\section{CONCLUSÃO}

O FDT mostrou-se útil para o rastreamento de glaucoma na população de funcionários da Santa Casa de Misericórdia de São Paulo. A inclusão de teste de avaliação funcional de fácil e rápida execução permite maior sensibilidade na detecção do glaucoma.

\section{ABSTRACT}

Purpose: To study the prevalence of glaucoma in employees of a public hospital in São Paulo and to analyze the role of frequency doubling perimetry along with non-contact tonometry and direct ophthalmoscopy, in the screening for glaucoma. Methods: 612 employees of the Central Hospital of the "Santa Casa de São Paulo" were evaluated from October 15 to 20, 2000; 438 were women and 174 men, with mean age of $45.05 \pm 7.7$ years (range, 35 to 81 years); 437 were white, 104 mulatto, 43 black and 28 Asian. All examinations were done by 10 eye specialists with experience in glaucoma. All employees had both eyes examined, by means of frequency doubling perimetry (FDT), ophthalmoscopy (FO), and non-contact tonometry (TNC). Results: 159 (25.98\%) subjects presented with at least one abnormal testing; 5 (3.14\%) had high IOP $(\mathrm{TNC}+) ; 13(8.17 \%)$ presented suspicious disk (FO+); 110 $(69.18 \%)$ presented abnormal FDT (FDP+); 8 (5.03\%) TNC+ and FO+; 10 (6.28\%) had TNC+ and FDT+; 9 (5.66\%) presented $\mathrm{FO}+$ and FDT+; 4 (2.51\%) had $\mathrm{TNC}+, \mathrm{FO}+$ and FDT + ; 12 (1.96\%) individuals were diagnosed with glaucoma, four of whom $(0.65 \%)$, with normal pressure glaucoma. Conclusions: Frequency doubling perimetry was found to be an important step in the screening for glaucoma in the study. Of the 12 individuals that had the diagnosis of glaucoma, 5 (41.6\%) would not have been diagnosed if they had not undergone frequency doubling perimetry.
Keywords: Glaucoma/diagnosis; Glaucoma/epidemiology; Perimetry/methods; Tonometry, ocular; Ophthalmoscopy

\section{REFERÊNCIAS}

1. Quigley HA. Number of people with glaucoma worldwide. Br J Ophthalmol. 1996;80(5):389-93. Comment In: Br J Ophthalmol. 1996;80(5):385-6.

2. Ivers RQ, Optom B, Macaskill P, Cumming RG, Mitchell P. Sensitivity and specificity of tests to detect eye disease in an older population. Ophthalmology. 2001;108(5):968-75.

3. Wilson MR, Khanna S. The value of different screening techniques for glaucoma. Curr Opin Ophthalmol. 1994;5(1):69-75.

4. Mundorf TK, Zimmerman TJ, Nardin GF, Kendall KS. Automated perimetry, tonometry, and questionnaire in glaucoma screening. Am J Ophthalmol. 1989;108(5):505-8. Comment in: Am J Ophthalmol. 1990;109(3):362-3.

5. Harper R, Reeves B. The sensitivity and specificity of direct ophthalmoscopic optic disc assessment in screening for glaucoma: a multivariate analysis. Graefes Arch Clin Exp Ophthalmol. 2000;238(12):949-55.

6. Michelson G, Groh MJ. Screening models for glaucoma. Curr Opin Ophthalmol. 2001;12(2):105-11.

7. Wu L, Suzuki Y, Kunimatsu S, Araiae M, Iwase A, Tomita G. Frequency doubling technology and confocal scanning ophthalmoscopic optic disc analysis in open-angle glaucoma with hemifield defects. J Glaucoma. 2001;10(4): 256-60.

8. Johnson CA. Recent developments in automated perimetry in glaucoma diagnosis and managements. Curr Opin Ophthalmol. 2002;13(2):77-84.

9. Serguhn S, Speigel D. Comparison of frequency doubling perimetry and standard achromatic computerized perimetry in patients with glaucoma. Graefes Arch Clin Exp Ophthalmol. 2001;239(5):351-5.

10. Anderson DR. Automated static perimetry. St. Louis: Mosby; 1993.

11. Tielsch JM, Katz J, Singh K, Quigley HA, Gottsch JD, Javitt J, et al. A population-based evaluation of glaucoma screening: the Baltimore Eye Survey. Am J Epidemiol. 1991; 134(10):1102-10.

12. Ralson ME, Choplin NT, Hollenbach KA, Applegate BJ, Henn TW. Glaucoma screening in primary care: the role of noncontact tonometry. J Fam Pract. 1992;34(1):73-7.

13. Vernon SA, Jones SJ, Henry DJ. Maximising the sensitivity and specificity of noncontact tonometry in glaucoma screening. Eye. 1991;5(Pt 4):491-3.

14. Cioffi GA, Mansberger S, Spry P, Spry P, Johnson C, Van Buskirk EM. Frequency doubling perimetry and the detection of eye disease in the community. Trans Am Ophthalmol Soc. 2000;98:195-9.

15. Johnson CA, Samuels SJ: Screening for glaucomatous visual field loss with frequency-doubling perimetry. Invest Ophthalmol Vis Sci 1997;38(2):413-25.

\title{
XIII SIMPÓSIO DE ATUALIZAÇÃO EM OFTALMOLOGIA
}

\section{1 o 02 de ABrin de 2005 Jurerê B. Village Hotel - Florianópolis - SC}

\begin{abstract}
Apoio: Associação Paranaense de Oftalmologia - APO Sociedade de O ftalmologia do Rio Grande do Sul - SORIGS Associação Catarinense de 0 ftalmologia - ACM
\end{abstract}

Informações: Praxis Feiras e Congressos

Tel.: (47) 326-1313 - HOME-PAGE: www.praxis.srv.br 